\title{
Three-Dimensional Pore Structure and Ion Conductivity of Porous Ceramic Diaphragms
}

\author{
Daniel Wiedenmann \\ Dept. of Geosciences and FRIMAT, University of Fribourg, Chemin du Musée 6, Pérolles, \\ Fribourg $\mathrm{CH}-1700$, Switzerland \\ Lukas Keller \\ EMPA, Swiss Federal Laboratories for Materials Testing and Research, \\ Überlandstrasse 129, Dübendorf CH-8600, Switzerland \\ Lorenz Holzer \\ ZHAW, Institute of Computational Physics ICP, Zurich University of Applied Sciences, \\ Wildbachstrasse 21, Winterthur CH-8400, Switzerland

\section{Jelena Stojadinović, Beat Münch, Laura Suarez, Benjamin Fumey, Harald Hagendorfer, and Rolf Brönnimann \\ EMPA, Swiss Federal Laboratories for Materials Testing and Research, Überlandstrasse 129, Dübendorf CH-8600, Switzerland}

\section{Peter Modregger} \\ Swiss Light Source, Paul Scherrer Institut, Villigen CH-5232, Switzerland \\ School of Biology and Medicine, University of Lausanne, Lausanne CH-1015, Switzerland
}

Michal Gorbar, Ulrich F. Vogt, and Andreas Züttel

EMPA, Swiss Federal Laboratories for Materials Testing and Research, Überlandstrasse 129, Dübendorf $\mathrm{CH}-8600$, Switzerland

\section{Fabio La Mantia}

CES, Center for Electrochemical Sciences, Ruhr-University Bochum, Universitätsstrasse 150, Bochum D-44780, Germany

\section{Roger Wepf}

ETH, Swiss Federal Institute of Technology Zurich, Electron Microscopy Centre EMEZ, Wolfgang-Pauli-Str. 16, Zurich $\mathrm{CH}-8093$, Switzerland

\section{Bernard Grobéty}

Dept. of Geosciences and FRIMAT, University of Fribourg, Chemin du Musée 6, Pérolles, Fribourg $\mathrm{CH}-1700$, Switzerland

The ion conductivity of two series of porous ceramic diaphragms impregnated with caustic potash was investigated by electrochemical impedance spectroscopy. To understand the impact of the pore structure on ion conductivity, the threedimensional (3-D) pore geometry of the diaphragms was characterized with synchrotron x-ray absorption tomography. Ion migration was calculated based on an extended pore structure model, which includes the electrolyte conductivity and geometric pore parameters, for example, tortuosity $(\tau)$ and constriction factor $(\beta)$, but no fitting parameters. The calculated ion conductivities are in agreement with the data obtained from electrochemical measurements on the 
diaphragms. The geometric tortuosity was found to be nearly independent of porosity. Pore path constrictions diminish with increasing porosity. The lower constrictivity provides more pore space that can effectively be used for mass transport. Direct measurements from tomographs of tortuosity and constrictivity opens new possibilities to study pore structures and transport properties of porous materials.

Keywords: constrictivity, diaphragm, ion conductivity, porosity, tortuosity, tomography

\section{Introduction}

$\mathrm{M}$ ass transport through porous media strongly depends on the structure of the pore network. Besides porosity, geometric aspects like the poresize distribution (PSD) and the connectivity of the pore network directly influence the mass transport properties of a porous material. Numerous authors ${ }^{1-9}$ experimentally investigated the dependency of mass transport through porous media on microstructure parameters. Nevertheless, the quantification of structure-related key parameters is accompanied with methodical difficulties. The interpretation of results obtained from experiments capturing transport properties on a macroscopic scale are often accounted for by fitting factors due to a lack of methodologies that enable quantification of the relevant microstructural features. However, recent progress in tomography and three-dimensional (3-D)-image processing opens new possibilities to study effects of tortuosity, constrictivity, PSDs and connectivity, which is the main focus of the present study.

Although the total porosity $\left(\varepsilon_{\text {tot }}\right)$ is defined as the fraction of the bulk volume of a porous sample that is occupied by void space, ${ }^{10}$ the effective porosity $\left(\varepsilon_{\mathrm{eff}}\right)$, that is, the pore volume that contributes to mass transport, can differ significantly from $\varepsilon_{\text {tot }}$. The difference may be due to isolated and blindended pores but also by constricted pore pathways (bottlenecks) reducing the effective pore space available for mass transport. Pore orientation and related path length are additional parameters, which influence the mass transport capabilities of a percolating pore system. Permeability- or conductivity-measurements enable the acquisition of bulk transport properties and average pore network parameters, but do not identify space resolved microstructural information.

Basic considerations regarding the relation of geometric aspects of the pore structure and mass transport are discussed by Carman. ${ }^{3}$ Based on Darcy's law and the findings of Blake $^{11}$ and Kozeny, ${ }^{6}$ Carman empirically derived a relation for the flow rate $(u)$ of a permeability measurement (Eq. 1)

$$
u=\frac{\varepsilon^{3}}{k \eta S^{2}} \frac{\Delta p}{L}
$$

(with $\Delta p$ : pressure difference, $\eta$ : fluid viscosity, $\varepsilon$ : porosity, $S$ : pore surface, and $L$ : sample length). Nevertheless, a theoretically derived factor $k$ (the Kozeny-constant) is necessary to correlate experimental results obtained from materials with different pore structures. The Kozeny constant is defined as the product of the square of tortuosity $(\tau)$ and a shape factor $\left(k_{0}\right)$, which depends on the morphology of the particles forming the porous medium ${ }^{3}$ (Eq. 2)

$$
k=\tau^{2} k_{0}
$$

The tortuosity factor $\tau$ is defined as the ratio of the effective pore pathway length $\left(l_{\text {eff }}\right)$ over the length of the sample $(L$; Figure 1$)$ in the direction of the bulk fluid flow ${ }^{3}$ (Eq. 3)

$$
\tau=\frac{l_{\mathrm{eff}}}{L}
$$

Thus, permeability measurements allow to determine the average transport capabilities of a sample provided the shape factor is known.

Based on the analogy between mass and electric transport along a pressure, respectively, electric potential, fluid-transfer properties of porous media (rocks, beds of particles) have also been determined electrochemically by ion conductivity measurements. ${ }^{1,2,4,5,7-9}$ An electric field across a porous sample soaked with a liquid electrolyte causes migration of ions and, thus, an electric current through a percolating pore network. In the case of a nonconductive porous medium, the measured ion conductivity shows ohmic behavior and depends on the transport capability of the electrolyte filled pore network. ${ }^{4,5,7}$ Wyllie and Rose ${ }^{9}$ proposed the derivation of Carman's tortuosity factor from conductivity measurements to determine the porosities of porous media from measured conductivities. Their underlying pore network

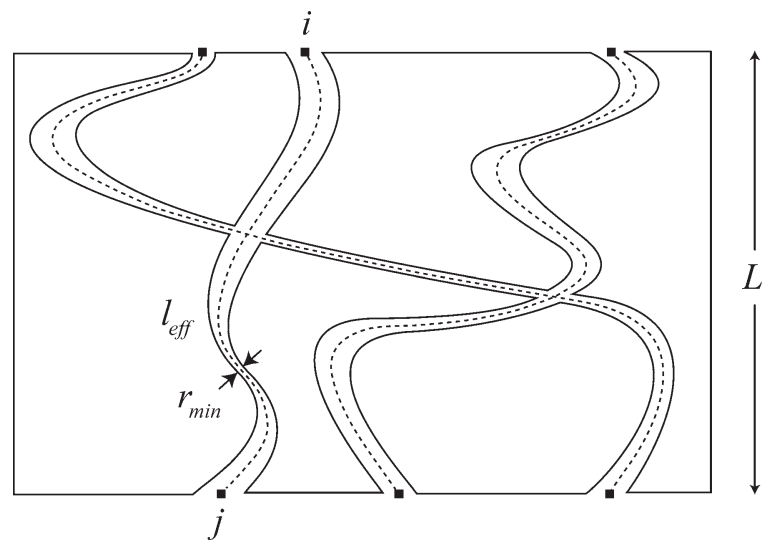

Figure 1. Schematic 2-D representation of the parameters characterizing a pore structure with three intersecting pore paths.

The dotted line connecting the entrance and exit points $i$ and $j$ has the length $l_{\text {eff. }}$ The sample has the length $L$ and geometric tortuosity $\tau_{\text {geo }}$ of a pore path is defined by the ratio $l_{\text {eff }} / L . \tau_{\text {geo }}$ and the minimal pore radius along the pore path (constriction) determine the mass transport through a porous system. 
geometry corresponds to the uniform tortuosity model. ${ }^{4,7,8}$ It describes all pore pathways as distinct circular tubes, which have the same length $\left(l_{\text {eff }}\right)$. The tortuosity $l_{\text {eff }} / L$ is thus considered to be the same for each pore path. In addition, individual pores have a constant diameter $(d)$ within a single pathway. For a nonconducting matrix, the electric conductance $(G)$ of a cylindrical porous sample filled with a liquid is inverse proportional to the sample length and proportional to the total cross-sectional pore area $\left(A_{\mathrm{p}}\right)$, which is the sum of the individual pore cross sections ${ }^{4}\left(\pi / 4 * \Sigma d^{2}\right)$ (Eq. 4). The conductivity of the liquid is $\sigma_{0}$

$$
G=\frac{\sigma_{0} \pi}{4 \tau L} \sum d^{2}
$$

The cross-sectional pore area $\left(A_{\mathrm{p}}\right)$ can be expressed by the product of porosity $(\varepsilon)$ multiplied with the total cross-sectional area $(A)$ of the porous cylindrical sample (Eq. 5)

$$
A_{\mathrm{p}}=\frac{\pi}{4} \sum d^{2}=\varepsilon A
$$

The combination of both equations allows for a term expressing the tortuosity factor $\tau$ as a function of $\varepsilon$ and the resistance $R$, which is called electric tortuosity $\tau_{\text {elc }}$. In this way, $\tau_{\text {elc }}$ is calculated without the determination of the cross-sectional pore area $\left(A_{\mathrm{p}}\right)$. In addition, by taking into account sample dimensions ( $A$ and $L), R$ can be replaced by the effective conductivity $\left(\sigma_{\text {eff }}\right)($ Eq. 6$)$

$$
\tau_{\mathrm{elc}}=\frac{\sigma_{0} R \varepsilon A}{L}=\frac{\sigma_{0}}{\sigma_{\mathrm{eff}}} \varepsilon
$$

or (Eq. 7)

$$
\varepsilon=\tau_{\text {elc }} \frac{\sigma_{\text {eff }}}{\sigma_{0}}
$$

It was obvious to the above authors that the permeabilityor conductivity-measurements only examine the transport property of a bulk without giving precise structural information on the pore network. $\tau_{\text {elc }}$ reflects only the true geometrically defined tortuosity $\left(\tau_{\text {geo }}\right)$ of samples for which the uniform tortuosity model is applicable. In all other cases, $\tau_{\text {elc }}$ captures all microstructure effects that influence the conductivity, not only tortuosity. Apparent variations of the "electrical tortuosity" may thus not only be due to changes of the geometric tortuosity but also of other stereological parameters, the most prominent being the variation of pore diameters along the trajectories of the pores (bottlenecks, constrictivity).

Most porous media cannot be described using a uniform tortuosity model. The relationship between conductivity and porosity for such media is thus not only depending on the tortuosity as deduced from Eq. 7. An empirical relationship between porosity and conductivity for such cases was given by Archie. ${ }^{1}$ He studied the transport properties in a series of rock samples that underwent the same sedimentation and diagenesis (solidification) history and found a power law dependency between normalized conductivity $(=1 / \mathrm{F}$ in Archie's formulation, with F called the "formation factor") and porosity (Eq. 8)

$$
\frac{\sigma_{\text {eff }}}{\sigma_{0}}=\varepsilon^{m}
$$

Archie observed that for samples with different porosities but similar microstructure or "fabrication history" (e.g., a series of rocks from the same geological formation or a series of mineral diaphragms with the same sintering treatment) a $\log (F)$ vs. $\log \left(\varepsilon_{\text {tot }}\right)$ plot showed a linear trend with slope $m$. Consequently, $m$ is an empirically derived formation- or series-specific constant. As for $\tau_{\text {elc }}$, the effects of the pore network parameters on conductivity other than porosity are contained in the exponent $m$. Archie's law, therefore, also captures the effect of microstructural changes between different sample series; however, it does not give any information, which features of the pore structure are relevant for mass transport.

One condition of the uniform tortuosity model, which is unusual in many real porous media, is the constant diameter along a pore trajectory. The diameter varies along the trajectory with the smallest diameter (constriction) being relevant for the transport properties. The effect of constrictions on conductivity has been calculated for a cylindrical tube with a hyperbolic pore neck. ${ }^{12}$ From the solution of the transport equations within this geometrical framework, it has been shown that the effect of the constriction is proportional to the ratio $\left(A_{\max } / A_{\min }\right)$, whereby $A_{\max }$ is the maximum crosssectional area at the entrance of the tube and $A_{\min }$ is the minimum cross-sectional area at the location of the tubes' constriction. This ratio has been called "constriction factor" $(\beta)$. For simplicity, in our work, we use the inverse ratio to obtain $\beta$-values between 0 and 1 (Eq. 9)

$$
\beta=\frac{A_{\min }}{A_{\max }}
$$

For cylindrical pore systems whose cross sections can be described with distinct radii at the constriction and the pipe entrance, the constriction factor is given by (Eq. 10)

$$
\beta=\frac{\pi r_{\min }^{2}}{\pi r_{\max }^{2}}
$$

Therefore, the constrictions along the pore trajectories reduce the conductivity. This reduction is taken into account by adding the constriction factor $(\beta)$ into Eq. 7 (Eq. 11)

$$
\frac{\sigma_{\text {eff }}}{\sigma_{0}}=\frac{\varepsilon \beta}{\tau_{\mathrm{geo}}}
$$

However, in the past, the transport relevant radii $\left(r_{\text {min }}\right.$; $\left.r_{\max }\right)$ and the associated $\beta$ value could not be determined for complex, disordered microstructures. Due to this lack of methodology, there is no experimental data, with which to validate the introduction of this constriction factor.

A direct way to obtain the topological key parameters is to measure them on reconstructed pore networks using 3-D tomographic imaging. The goal of this work was to compare calculated conductivities (based on the tomographically determined stereological parameters and Eq. 11) with measurements obtained from conductivity experiments. The ceramic diaphragms used for this purpose have been prepared 
Table 1. Summary of Quantitative Results for Various Parameters That Were Determined in This Study

\begin{tabular}{|c|c|c|c|c|c|c|c|c|c|c|c|c|}
\hline Sample & $\begin{array}{c}\sigma(\mathrm{S} / \mathrm{cm}) \\
\text { EIS }\end{array}$ & $\begin{array}{c}\sigma_{\beta}(\mathrm{S} / \mathrm{cm}) \\
\text { tomo }\end{array}$ & $\begin{array}{c}F \\
\text { Archie }\end{array}$ & $\begin{array}{c}m \\
\text { Archie }\end{array}$ & $\begin{array}{l}\tau_{\text {elc }} \\
\text { EIS }\end{array}$ & $\begin{array}{l}\delta_{\text {elc }} \\
\text { EIS }\end{array}$ & $\begin{array}{c}\tau_{\text {geo }} \\
\text { tomo }\end{array}$ & $\begin{array}{c}r_{\min }(\mu \mathrm{m}) \\
\mathrm{MIP}\end{array}$ & $\begin{array}{c}r_{\max }(\mu \mathrm{m}) \\
\mathrm{cPSD}\end{array}$ & $\begin{array}{c}\beta_{\text {geo }} \\
\text { tomo }\end{array}$ & $\begin{array}{c}\varepsilon_{\text {tot }} \\
\text { tomo }\end{array}$ & $\begin{array}{l}\varepsilon_{\text {eff }} \\
\text { EIS }\end{array}$ \\
\hline $\mathrm{KOH}$ & 0.645 & - & 1.00 & 1.00 & 1.00 & - & 1.00 & - & - & - & 1.00 & 1.00 \\
\hline ol 1 & 0.031 & 0.040 & 20.98 & 2.31 & 5.61 & 0.32 & 1.82 & 1.87 & 2.87 & 0.42 & 0.27 & 0.09 \\
\hline ol 2 & 0.047 & 0.052 & 13.72 & & 4.42 & 0.42 & 1.87 & 2.57 & 3.74 & 0.47 & 0.32 & 0.14 \\
\hline ol 3 & 0.074 & 0.092 & 8.70 & & 3.63 & 0.49 & 1.77 & 3.74 & 4.80 & 0.61 & 0.42 & 0.20 \\
\hline ol 4 & 0.106 & 0.093 & 6.11 & & 2.71 & 0.64 & 1.74 & 4.32 & 5.74 & 0.57 & 0.44 & 0.28 \\
\hline wo 1 & 0.063 & 0.085 & 10.24 & 2.95 & 4.63 & 0.38 & 1.76 & 2.40 & 3.30 & 0.53 & 0.45 & 0.17 \\
\hline wo 2 & 0.092 & 0.099 & 7.04 & & 3.67 & 0.47 & 1.74 & 2.98 & 4.02 & 0.55 & 0.52 & 0.25 \\
\hline wo 3 & 0.140 & 0.143 & 4.62 & & 2.71 & 0.60 & 1.62 & 3.71 & 4.52 & 0.67 & 0.59 & 0.35 \\
\hline wo 4 & 0.254 & 0.186 & 2.54 & & 2.02 & 0.91 & 1.84 & 9.14 & 11.51 & 0.63 & 0.80 & 0.73 \\
\hline
\end{tabular}

$\sigma$ : conductivity of soaked diaphragms (from measurements with EIS and calculated based on tomographic parameters); F: Archie's formation factor; $m$ : seriesspecific constant derived from Archie's law; $\tau_{\text {elc }}$ : electric tortuosity; $\delta_{\text {elc }}$ : electric constrictivity; $\tau_{\text {geo }}$ : geometric tortuosity; $r_{\text {min }}$ : pore radius corresponding to 50 vol $\%$ of the MIP-PSD; $r_{\max }$ : pore radius corresponding to $50 \mathrm{vol} \%$ of the c-PSD; $\beta_{\text {geo }}$ : constriction factor; $\varepsilon_{\text {tot }}$ : total porosity; $\varepsilon_{\text {eff }}$ : effective porosity.

in the framework of a project investigating substitutes for chrysotile-asbestos gas separation diaphragms applied in alkaline electrolysis. The industrial use of chrysotile-asbestos is forbidden in many countries and manufacturers of electrolyseurs are forced to replace the asbestos diaphragms. Two sets of diaphragms, one made of olivine and the other of wollastonite, with distinct pore structure architectures covering a wide range of porosities were analyzed. 3-D reconstructions of the pore network were calculated from synchrotron radiation $\mathrm{x}$-ray tomography. The quantification of the pore structure was done using modern 3-D image analysis methods and the ion conductivity of the same diaphragms was measured using electrochemical impedance spectroscopy (EIS). The transport properties are discussed using the directly determined stereological parameters.

\section{Experimental}

\section{Diaphragm fabrication}

Two series of mineral-based ceramic diaphragms with varying porosity (Table 1) were fabricated by sintering. Green bodies were prepared from mixtures of olivine (provided by North Cape Minerals, Krefeld, Germany) or wollastonite (provided by Mial, Feldmeilen, Switzerland) powder with different proportions of a carbon pore former (Sigradur ${ }^{\circledR}$ carbon powder, HTW Germany) and dissolved polyvinylalcohol (Optapix PA 4 G, Zschimmer \& Schwartz, Germany, 20 wt $\%$ water based) as binder. The mixtures were thoroughly homogenized by ball milling and uniaxially pressed at $20 \mathrm{MPa}$. The diaphragms have a diameter of $50 \mathrm{~mm}$ and a thickness of approximately $4 \mathrm{~mm}$. Subsequently, the samples were sintered for $1 \mathrm{~h}$ at $1300^{\circ} \mathrm{C}$. The heating- and cooling-rates for reaching the sintering temperature were 1 and $3 \mathrm{~K} / \mathrm{min}$, respectively.

\section{Ion conductivity}

The transport properties of the porous diaphragms soaked with a concentrated $\mathrm{KOH}$ electrolyte were electrochemically determined with a differential resistance measurement method $^{13}$ using impedance spectroscopy (EIS). The sintered disks were reduced to a diameter of $20 \mathrm{~mm}$ and to a thickness of $2.5 \mathrm{~mm}$ by grinding and polishing. To completely saturate the free pore space, the diaphragms were progressively soaked with 25 wt \% caustic potash.

EIS experiments were performed with a four-electrode electrochemical cell in the frequency range from $100 \mathrm{kHz}$ to
$0.1 \mathrm{~Hz}$ in potentiostatic mode using a Zahner IM6eX potentiostat. The distance between the reference electrode and the sense electrode was $64 \mathrm{~mm}$ without diaphragm. The applied potential between the sense and reference electrodes was $0.75 \mathrm{~V}$, to which an AC signal with amplitude of $10 \mathrm{mV}$ was added. A detailed description of the experimental setup is published elsewhere. ${ }^{14}$

The four-electrode setup offers the possibility to separate the contribution of the diaphragm from the complex impedance of the anode and cathode, thus avoiding influences of double-layer capacitances at the interfaces between the electrolyte and the electrodes on the measured values. Applying an alternating current further helps to minimize such influences. For porous media made of electrically insolating materials, the impedance spectra are expected to be composed only by the in-phase part Z' (real part)., ${ }^{4,7}$ Consequently, the purely ohmic resistance of the diaphragm can be measured using reference and sense electrodes.

\section{3-D microstructure analysis}

Synchrotron radiation $x$-ray absorption tomography. For tomographic measurements, the sintered diaphragms were cold impregnated with the polymer system AralditBY158/ Aradur21 (supplied by Huntsman) to completely fill up the interconnected void space of the porous structure. The initial pressure of 20 mbar was increased to 2 bar after 50 min to allow the polymer to penetrate deeper into the pores. The impregnated samples were kept for $12 \mathrm{~h}$ at $80^{\circ} \mathrm{C}$ to completely cure the polymer. Finally, cylindrical samples with a diameter of $700 \mu \mathrm{m}$ were prepared by laser cutting.

Synchrotron radiation x-ray absorption tomography was performed at the Tomcat beamline at the Swiss Light Source at the Paul Scherrer Institute in Villigen, Switzerland. ${ }^{15}$ The absorption experiment was done with an x-ray energy of $15.5 \mathrm{keV}$ and a field of view of $0.78 \mathrm{~mm}$. Image stacks of 2048 slides with a resolution of $2048 \times 2048$ pixels and a pixel resolution of $370 \mathrm{~nm}$ were recalculated from 1601 projections. The reconstructed 2-D cross sections for the end members of the diaphragms series are shown in Figure 2.

Phase segmentation. For the segmentation of the 3-D structures and for image processing, the program Avizo was used. Image stacks from tomography were directly transformed into a 3-D data volume composed of $2048 \times 2048$ $\times 2048$ voxels, which results in a voxel resolution of 370 $\mathrm{nm}$. To avoid memory problems during computation of 


\section{olivine}
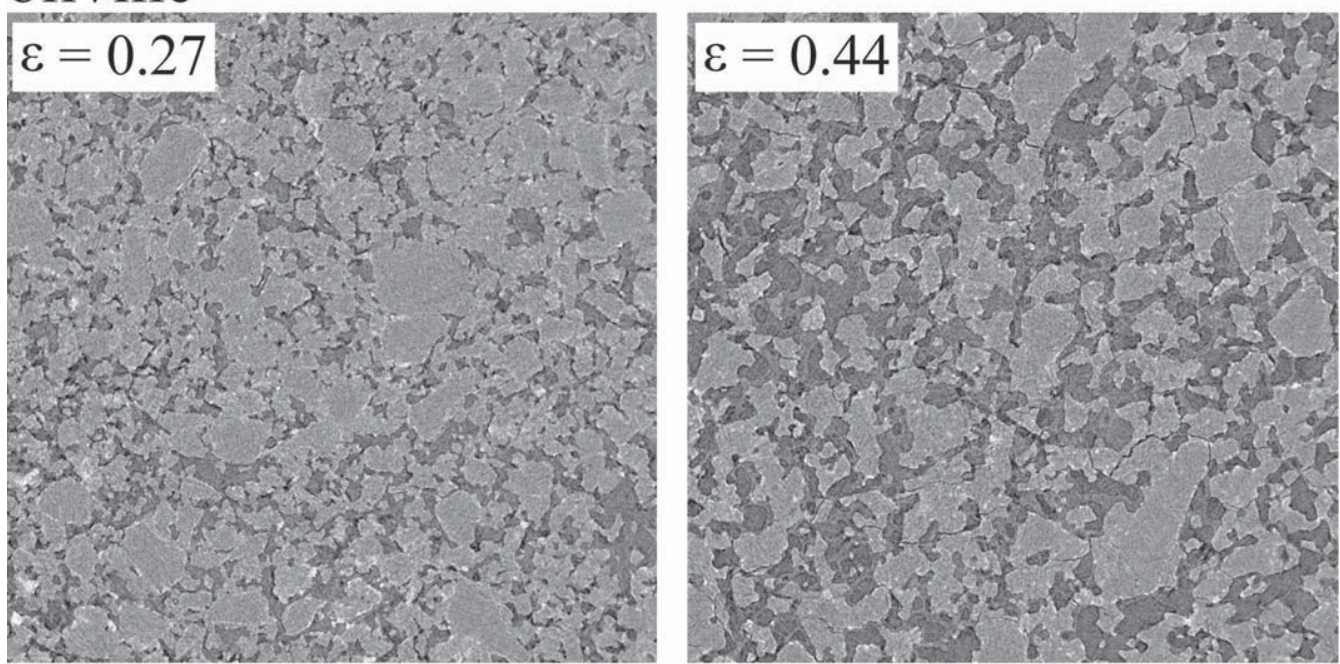

\section{wollastonite}
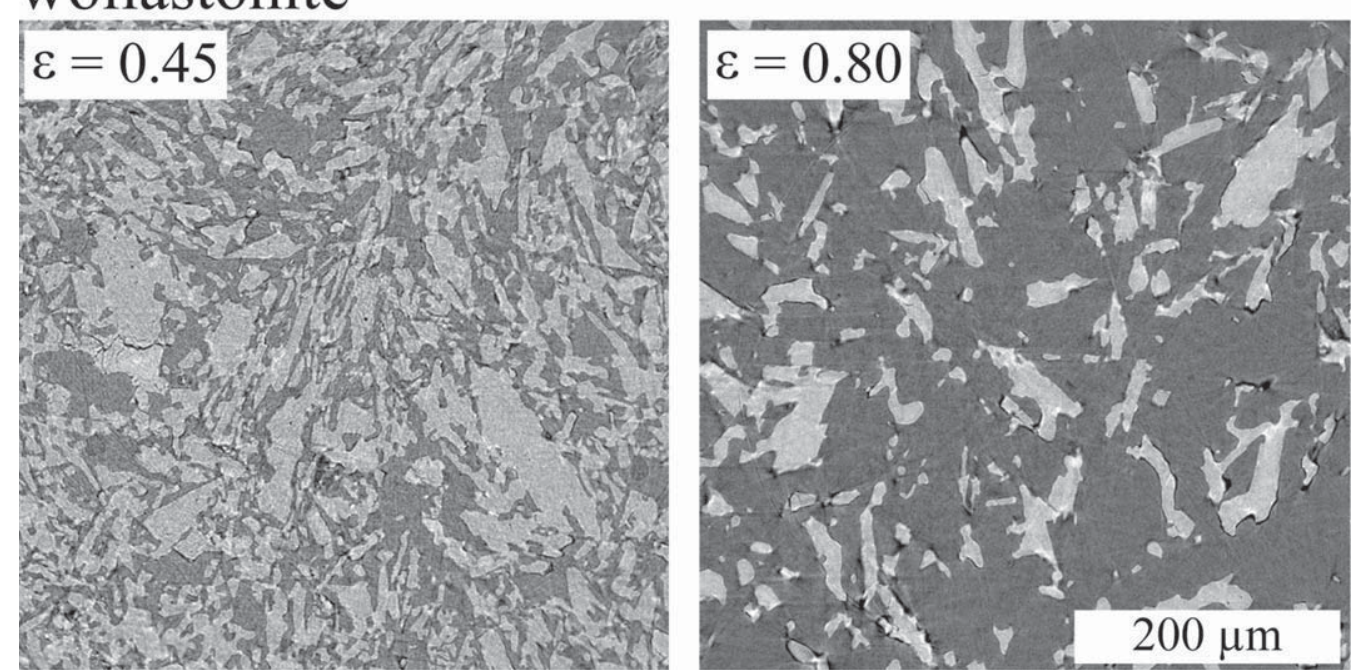

Figure 2. Reconstructed 2-D cross sections from x-ray absorption tomography of investigated mineral diaphragms (end members ol 1, ol 4, wo 1, and wo 4).

The cross sections are oriented perpendicular to the migration direction ( $z$ direction) of the ion conductivity experiment. Bright phase: particles (upper row: isometric olivine; lower row: fibrous wollastonite), dark phase: pores filled with epoxy.

topological parameters, cubic subvolumes of the acquired tomographs were cropped. After downsampling and applying a median filter for noise reduction, the reconstructed structures were binarized by 3-D thresholding. The final subvolumes have a cubic dimension of $660 \times 660 \times 660$ voxels and a voxel size of $740 \mathrm{~nm}$.

As the grain boundaries between particles and pores in the reconstruction are not sharp, but characterized by a diffuse greyscale gradient, segmentation by 3-D thresholding can represent a significant source of errors in the structural quantification using computational image processing. ${ }^{16}$

Continuous PSD and simulation of mercury intrusion porosimetry. PSDs were extracted from the reconstructed and segmented pore volume by using image processing techniques developed by Münch and Holzer. ${ }^{17}$ Two types of PSDs are obtained: the classical mercury intrusion porosimetry (MIP)-PSDs, which are affected by constrictions, and the so-called continuous c-PSDs, which represent nonconstricted pore dimensions.

The classical MIP-PSD is obtained in physical experiments by measuring the differential pore volume, which is filled with mercury for an incremental increase of the intrusion pressure. It is well-known that the MIP is affected by the bottleneck effect. ${ }^{18}$ During migration of mercury through the pore paths, the bulges of relatively large pores are filled after passing through narrow pore necks. Thereby the volumes of the larger pores are erroneously attributed to the radii of the pore necks and hence the small pores are overestimated. In physical experiments as well as in MIP simulations, this effect leads to a shift of the PSD to lower pore radii. The peak of the MIP-PSD is thus not the real average pore diameter, but represents rather the average diameter/ 


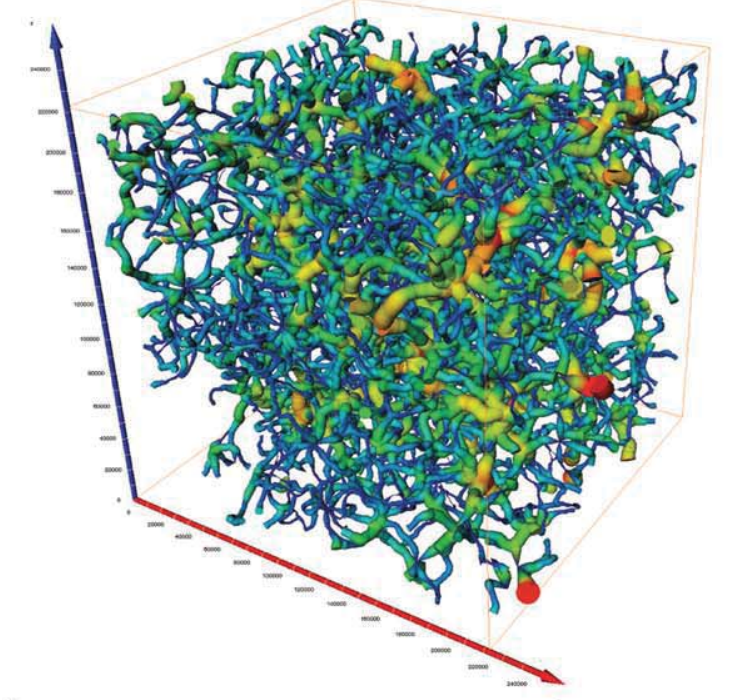

a

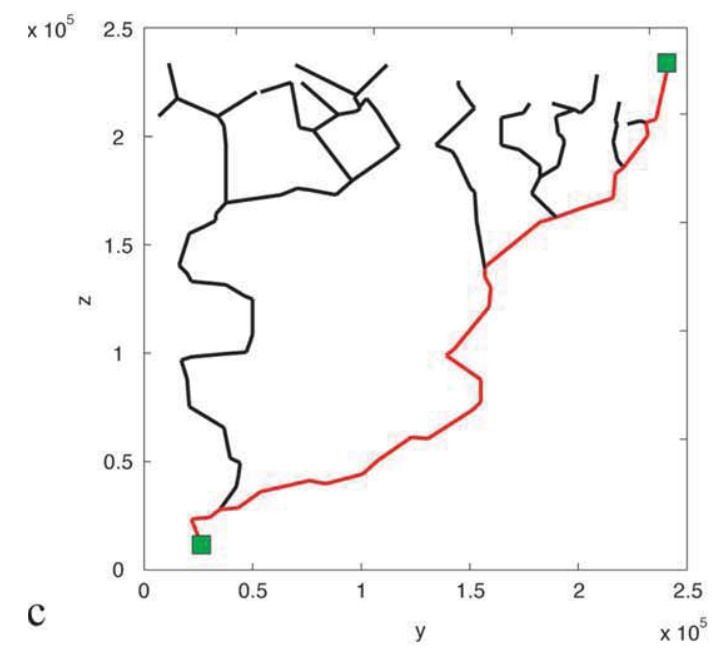

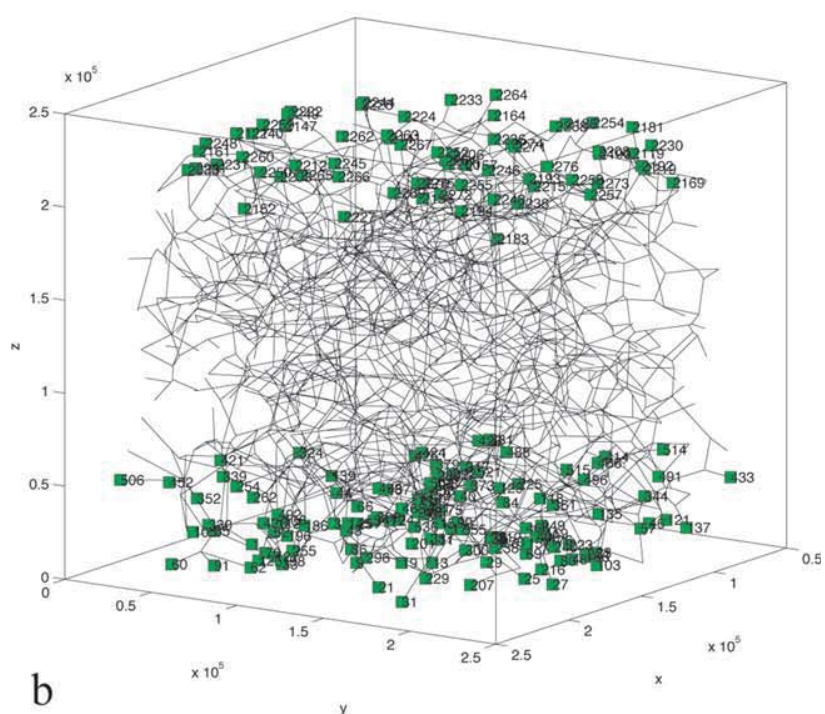

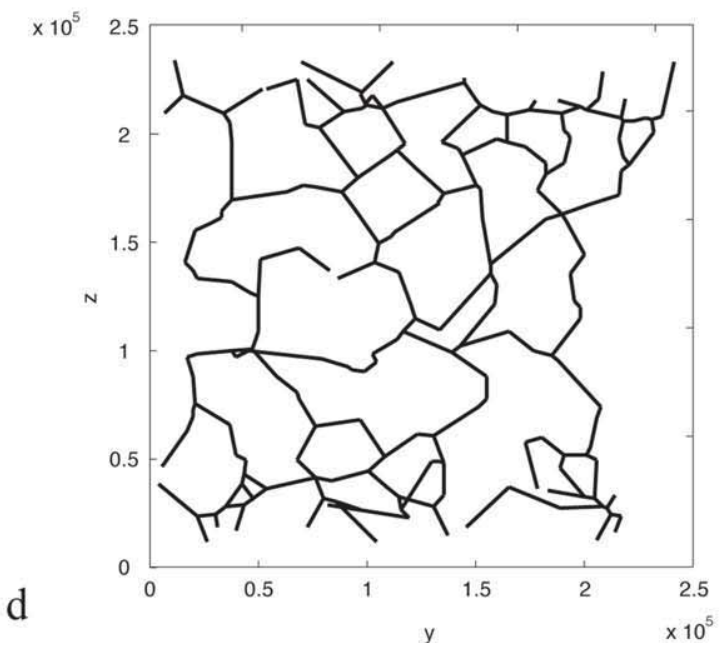

Figure 3. Voxel skeleton representing the pore space of a percolating pore system.

(a) 3-D section of a sample. The thickness and color of the segments reflects the pore radii. (b) Medial axis line skeleton of the pipe segments shown in Figure 3a. Green squares mark source and sink nodes within the $z$ bounding faces. (c) 2-D section of the line skeleton. The red path is the shortest connection between a pair of source and sink nodes. The ratio between the path length and the length of the sample cubical is defined as the geometric tortuosity of the pore path. Black paths correspond to all paths between one source node and all other sink nodes. (d) Shortest path network connecting all pairs of source and sink nodes in the $z$ planes.

radius of the bottlenecks, called the break through diameter or the constricted radius $r_{\text {min }}$ (see Eq. 10).

In contrast to the classical MIP measurements, the bottleneck effect is suppressed in the simulations of c-PSDs as explained in more detail in Münch and Holzer. ${ }^{17}$ The c-PSD measurement is based on a distance map, which is initiated at numerous seed points inside the pore network. Consequently, the pore necks (local minima) are approached from both sides. In this way, the necks have no artificial effect on the measured c-PSD and the measured size distributions reflect the nonconstricted dimensions at the pore bulges $\left(r_{\max }\right)$.

In the present study, the MIP- and c-PSD were used to determine the constriction factor $\left(\beta_{\text {geo }}\right)$. From the two types of PSD-curves, the $r_{50}$ radii corresponding to the 50 vol $\%$ fractiles (i.e., radius at $50 \%$ cumulative volume) are extracted and considered as average values which are characteristic for the pore structure dimensions. Explicitly the $r_{50}$ from the constricted MIP-PSD is taken as the average radius of the pore necks $\left(r_{\text {min }}\right)$, whereas the $r_{50}$ from the c-PSD curve is taken as the average radius at pore bulges $\left(r_{\max }\right)$. In this way, for each sample, a constriction factor $\left(\beta_{\text {geo }}\right)$ can be derived directly from the tomographs. Reproducibility tests have shown that the standard deviation of pore volume and $r_{50}$ determined by this method is $4-10 \%$ and in most cases below $5 \% .{ }^{19}$

Geometric tortuosity $\left(\tau_{\text {geo }}\right)$. To quantify the geometric tortuosity of the investigated samples, the pore path length 
Table 2. Average Values of Geometric Tortuosities $\left(\tau_{\text {geo }}\right)$ in the $z$ Direction, Amount of Considered Pore Paths $(n)$ and Standard Deviations (SD) for Investigated Diaphragms

\begin{tabular}{lccc}
\hline Sample & $\tau_{\text {geo }}$ & $n$ & SD \\
\hline ol 1 & 1.82 & 398522 & 0.17 \\
ol 2 & 1.87 & 298991 & 0.17 \\
ol 3 & 1.77 & 193610 & 0.17 \\
ol 4 & 1.74 & 129890 & 0.16 \\
wo 1 & 1.76 & 419079 & 0.16 \\
wo 2 & 1.74 & 303893 & 0.15 \\
wo 3 & 1.62 & 196060 & 0.10 \\
wo 4 & 1.84 & 60269 & 0.24 \\
\hline
\end{tabular}

of the reconstructed tomographs were determined by means of 3-D image analysis. Pore skeletons were extracted with the program Avizo (Figure 3). The length of the pore pathways in the skeletonized structures were then analyzed with a code, which was implemented in Matlab (as described below). Because bulk ion migration through the diaphragms occurs in $z$ direction of the tomographs, the calculations were done for the pore paths connecting the faces bounding the observed volume in $z$ direction. ${ }^{20}$

The determination of the geometric tortuosity is based on graph theory. ${ }^{21}$ As initial step, the complex pore volume, which in terms of image analysis is represented by a subset of voxels, is transformed into a voxel skeleton (Figure 3a). A skeleton consists of tube segments that are interconnected at nodes (intersection of two or more segments) and together they form a 3-D network. Establishing the tube segment medial axes allows the voxel skeleton to be converted into a line skeleton (Figure 3b).

Subsequently, the shortest path between a source node and a sink node in the direction of ion migration is identified by means of the Dijkstra's algorithm, ${ }^{22}$ and the path length is established by vector calculation (Figure 3c). The source and sink nodes are defined by the intersection of tube segments with the boundary faces of the analyzed volume. The number of shortest paths associated with a source node is equal to the number of sink nodes at the opposite boundary face. All pairs of source and sink nodes in the direction of mass transport are considered (Figure 3d). Shortest path tortuosities are then given by the ratio of the effective length of a shortest path $\left(l_{\text {eff }}\right)$ and the length of the sample cubical $(L)$ (Figure 3c). Finally, the average value of all determined tortuosities is calculated (Table 2).

\section{Results and Discussion}

\section{Porosity, geometrical tortuosity, and constrictivity}

The main microstructural parameter, which affects the transport properties of the analyzed samples, is porosity. Microstructures of the investigated porous diaphragm series acquired by $\mathrm{x}$-ray absorption tomography are illustrated in Figure 2. The mineral particles show a random distribution.
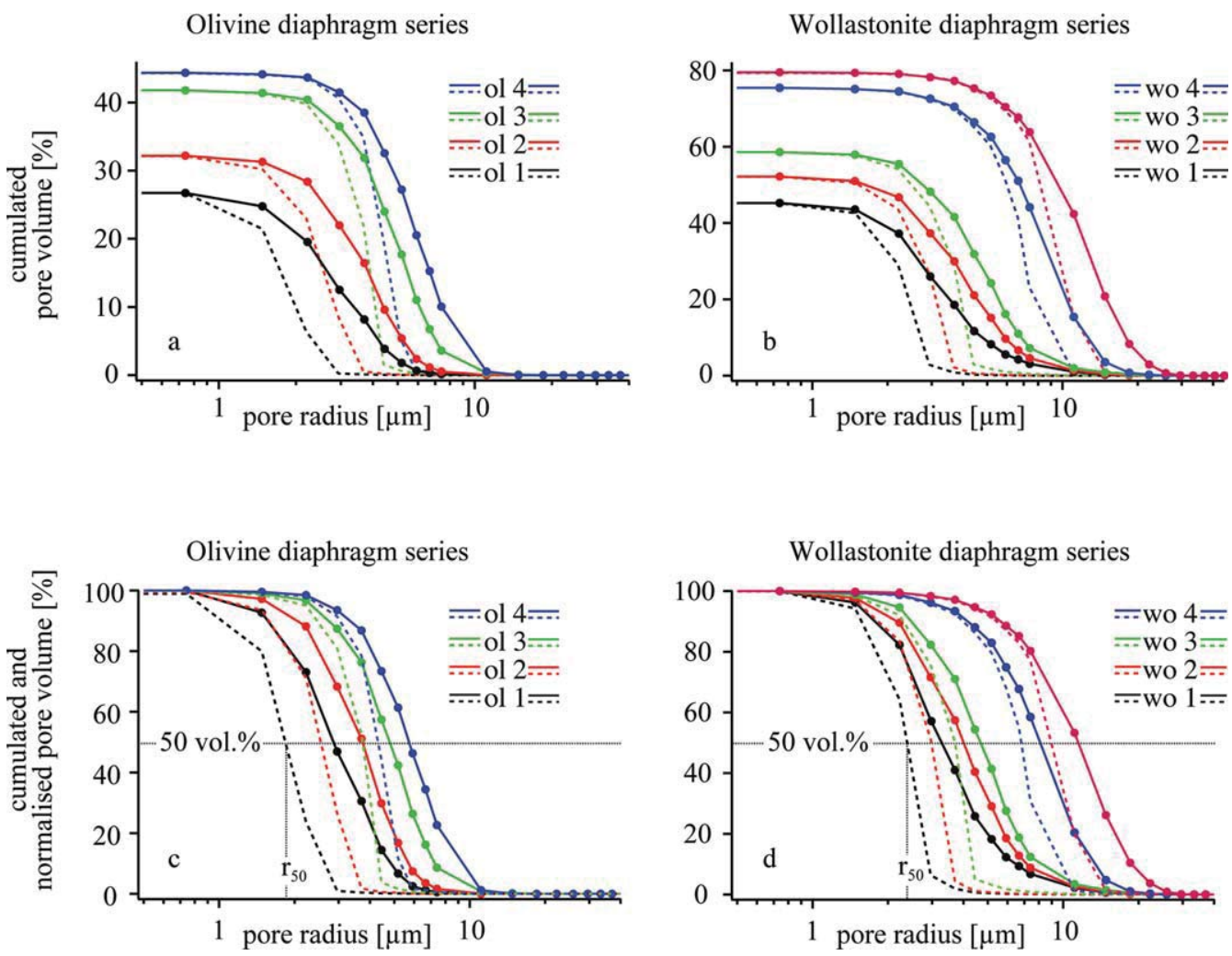

Figure 4. Tomography-based c-PSD(solid lines) and simulated MIP-PSD(dashed lines) of investigated diaphragms.

Olivine- (a) and wollastonite-diaphragms (b). PSDs normalized to $100 \%$ for olivine (c) and wollastonite (d). The curves are determined according to the pore size concept of Münch and Holzer (2008). 

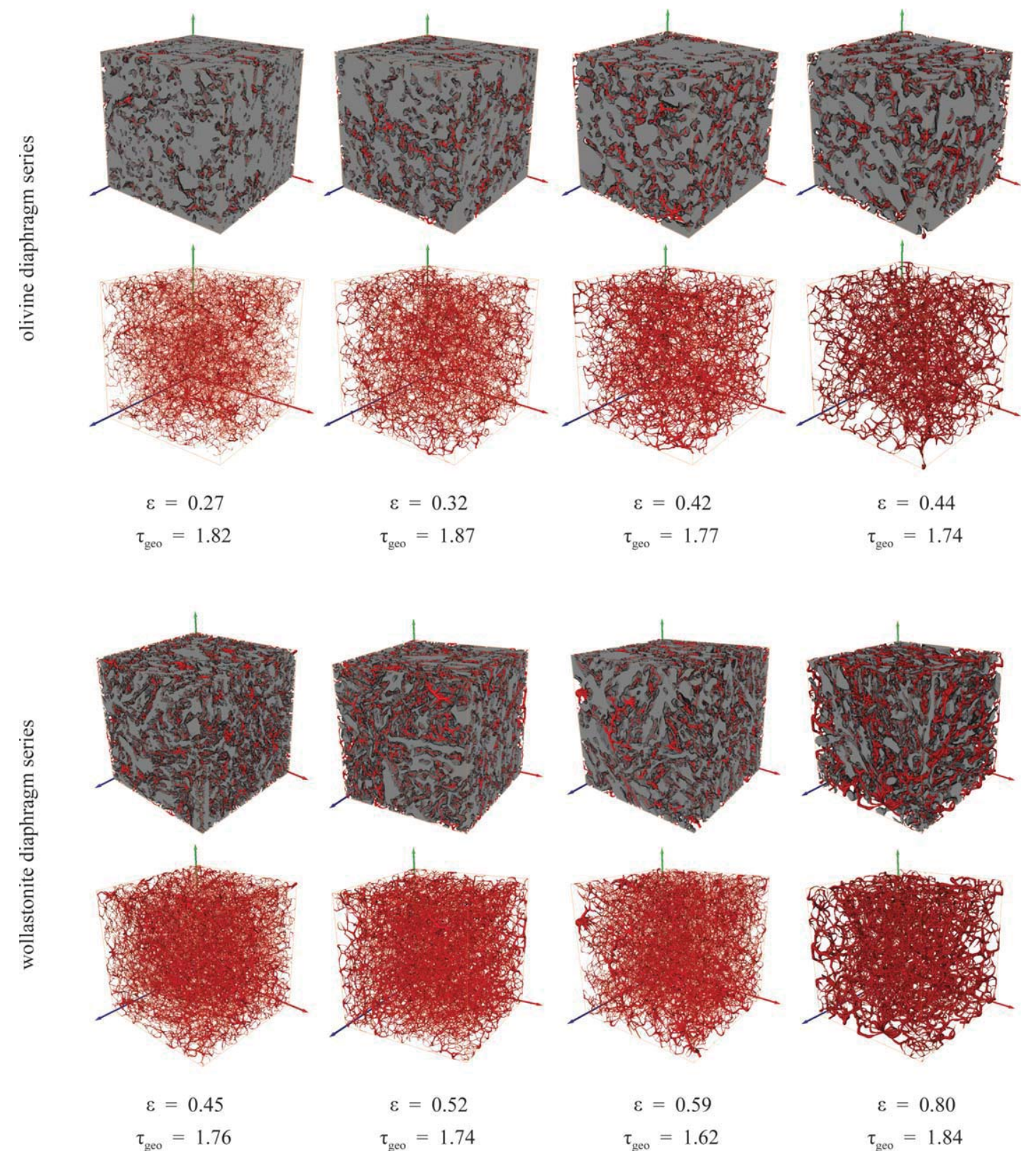

$$
\begin{aligned}
\varepsilon=0.45 & \varepsilon=0.52 \\
\tau_{\text {geo }}=1.76 & \tau_{\text {geo }}=1.74
\end{aligned}
$$$$
\varepsilon=0.59
$$$$
\tau_{\text {geo }}=1.62
$$

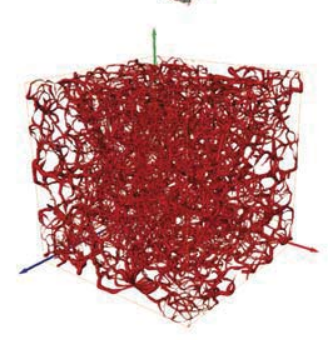

Figure 5. Reconstructed sample volumes and 3-D pore path visualization of the diaphragm series (based on $\mathbf{x}$-ray absorption tomography, edge length: $488 \mu \mathrm{m}$ ).

Porosities increase from left to right. For all investigated diaphragms, the geometric tortuosities are nearly constant. Mineral particles show a random distribution.

It is well-known that the shape and habitus of the mineral grains influence the geometry of the pores, ${ }^{2,3}$ that is, in the diaphragms made of rounded olivine grains the pores are isometric, whereas in the diaphragms made of acicular wollastonite grains they tend to be elongated.

The c-PSD derived porosity $\varepsilon$ varies from 0.27 to 0.44 for olivine, and from 0.45 to 0.80 for wollastonite, as can be seen from the c-PSD curves ( $y$ axis of Figures. $4 \mathrm{a}, \mathrm{b}$ and Table 1). The dashed lines in the figures represent the PSDs from mercury intrusion (MIP) simulations. The fact that the intrusion simulations result in the same total porosities indicates entirely percolating pore structures, that is, there are no isolated pores. For both series, the average pore radii increase with porosity. Due to the isometric habitus of the olivine crystals, no diaphragms with $\varepsilon_{\text {tot }}>0.44$ could be manufactured. Samples with higher porosities disintegrated during the sintering process. In contrast, the fibrous habitus of wollastonite allows for fabrication of samples with porosities up to 0.80 .

As described earlier, the geometric tortuosity can be extracted based on the skeleton network structure derived from tomographs (see Figure 3). A 3-D pore path visualization of the tomographically acquired diaphragm structures is shown in Figure 5. Analyzed samples show tortuosities that 

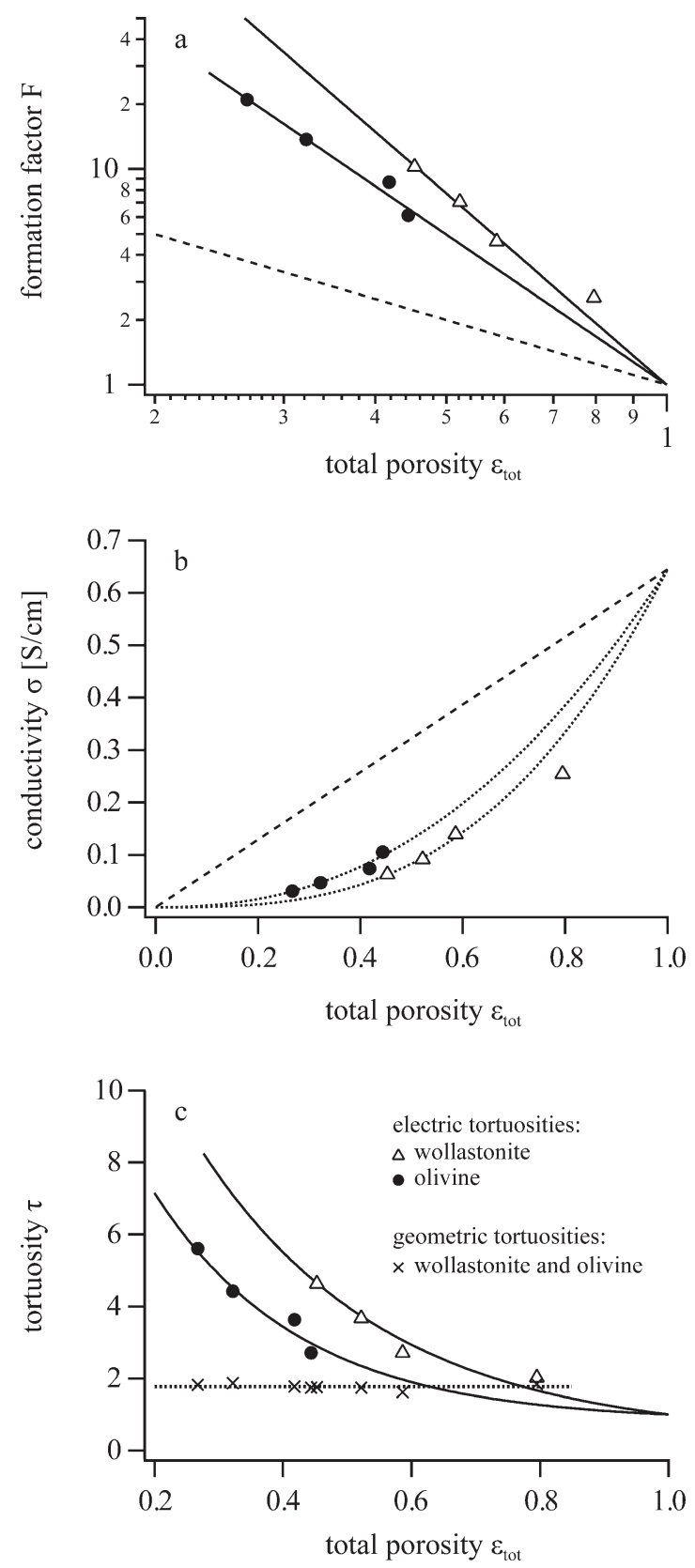

Figure 6. (a) Archie's formation factor F vs. total porosity $\varepsilon_{\text {tot }}$ (filled circles for olivine, open triangles for wollastonite).

The dashed line represents a hypothetical series of samples with straight tubes parallel to the applied field and equal diameter (no constriction) as pores, that is, samples for which $m=1$. (b) Conductivities measured by EIS for both diaphragm series vs. the corresponding total porosity. The dotted trend lines are fitted according to Archie's law ${ }^{1}$. (c) Electric and geometric tortuosities of the diaphragm series vs. the total porosity $\varepsilon_{\mathrm{tot}}$.

are clustered around a single mean value (Tables 1 and 2). Surprisingly, for both diaphragm series, no significant variation of $\tau_{\text {geo }}$ is observed over the entire range of porosity. All measured values are within a narrow range between 1.62 and 1.87 .

Cumulated PSDs normalized to $100 \%$ allow for a better comparison of the average pore sizes of the different samples (in contrast to the nonnormalized PSDs in Figures 4a and b). For each diaphragm, the $r_{\max }$ and $r_{\min }$ are determined by extracting the $r_{50}$ of the nonconstricted c-PSD (solid lines) and the $r_{50}$ of the constricted MIP-PSD (dashed lines), respectively (Figures $4 \mathrm{c}$ and $\mathrm{d})$. The ratio $\left(r_{\min } / r_{\max }\right)$ then gives the constriction factor $\left(\beta_{\text {geo }}\right)$, which is shown for each sample of the olivine- and wollastonite-series in Table 1. The results indicate that the constriction factor $(\beta)$ varies systematically as a function of porosity $(\varepsilon)$, unlike the geometric tortuosity $\left(\tau_{\text {geo }}\right)$, which is nearly constant.

\section{Stereological parameters and conductivity}

Archie's "formation" factors (= reciprocal normalized conductivities, Table 1) of the sintered olivine and wollastonite diaphragms define almost perfect linear trends in a $\log (\mathrm{F})$ vs. $\log \left(\varepsilon_{\mathrm{tot}}\right)$ plot with the slope $m=2.31$ for the olivine series and $m=2.95$ for the wollastonite series (Table 1, Figure $6 \mathrm{a}$ ), that is, for a given $\varepsilon_{\text {tot }}$, an olivine diaphragm has a higher conductivity than the corresponding wollastonite diaphragm. This is also reflected in the nonlinear correlation between ion conductivity and total porosity (Figure 6b). The trend lines of both series can be fitted according to Eq. 8 (dotted lines in Figure 6b).

The relationships between porosity and $\tau_{\text {elc }}$, respectively, $\tau_{\text {geo }}$ is illustrated in Figure $6 \mathrm{c}$. The electric tortuosity $\tau_{\text {elc }}$ increases exponentially with decreasing porosity. Thereby, both series follow different trend lines, which can be fitted by a logarithmic relationship derived by Ref. 23 . For a hypothetical porosity of $100 \%$, both trends give a value, which is close to the observed geometrical tortuosity (i.e., close to 1.77). For the wollastonite sample with the highest porosity, the measured $\tau_{\text {elc }}$ and $\tau_{\text {geo }}$ are almost identical.

Using the measured constriction factor and Eq. 11, allows calculating the conductivity from parameters entirely derived from tomography (Figure 7). The calculated values reproduce the measured values remarkably well (Table 1). The excellent correspondence between measured and calculated conductivity (Table 2) is a strong indication that electric transport across the investigated olivine and wollastonite diaphragms is only controlled by the intrinsic conductivity of

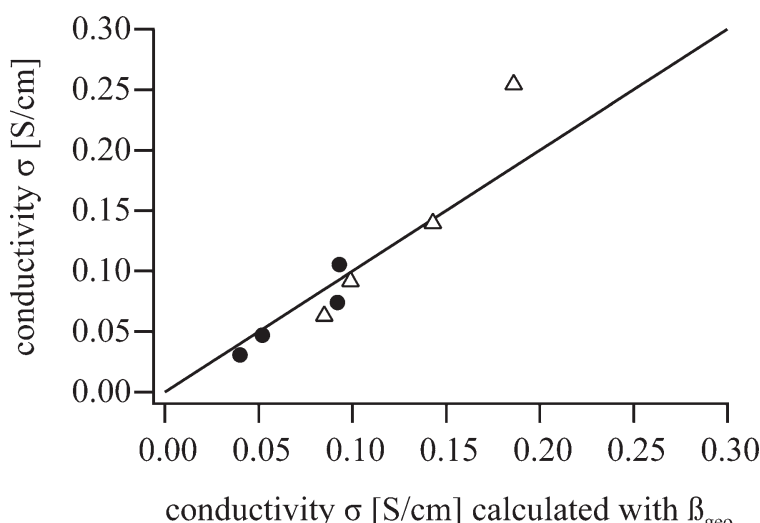

Figure 7. Effective conductivity $\sigma_{\text {eff }}$ measured by EIS vs. calculated conductivities from tomographic investigations (filled circles for olivine, open triangles for wollastonite). 
the electrolyte and the three stereological parameters porosity, tortuosity, and constrictivity.

The electrical constrictivity $\left(\delta_{\text {elc }}\right)$ can be obtained indirectly by substituting in Eq. 11 the experimentally determined conductivity $\left(\sigma_{\text {eff }}\right)$ and the structurally determined tortuosity $\left(\tau_{\text {geo }}\right)$ and porosity $\left(\varepsilon_{\text {tot }}\right)$, which results in the following expression (Eq. 12)

$$
\delta_{\mathrm{elc}}=\frac{\sigma_{\mathrm{eff}} \tau_{\mathrm{geo}}}{\sigma_{0} \varepsilon_{\mathrm{tot}}}
$$

The relevance of the electrical constrictivity for structural investigations can be questioned in the same way as for the electrical tortuosity, because both are determined indirectly and may, therefore, not reflect a single specific microstructure feature. It is shown in Figure 8a that in both diaphragm series the constrictivity $\left(\delta_{\text {elc }}\right)$ varies systematically with porosity. Thereby the constrictivities of the olivine- and the wollastonite-samples again follow two separate trends, which indicate that the structural differences between the two diaphragm series are also reflected by the different average pore neck dimensions determined with Eq. 12. By applying Archie's law, the trends for both diaphragm series are defined as (Eq. 13)

$$
\delta_{\text {elc }}=\tau_{\text {geo }} \varepsilon_{\text {tot }}^{\mathrm{m}-1}
$$

Because the geometric tortuosity is almost constant for both diaphragm series, the porosity, at which the constrictivity becomes one, that is, where transport is not affected by bottlenecks, can be calculated (Eq. 14)

$$
\varepsilon_{\max }=\tau^{\frac{1}{1-\mathrm{m}}}
$$

For the olivine- and wollastonite-series, these porosities are 0.65 and 0.75 , respectively. As shown in Figure 8a, the wollastonite sample with the highest porosity is very close to this apparent maximum value and has a constrictivity close to 1 .

\section{Effective porosity}

Constrictions due to pore necks limit the effective pore space available for mass transport. ${ }^{4}$ The effective porosity $\varepsilon_{\text {eff }}$ can be defined as the product of porosity $\left(\varepsilon_{\text {tot }}\right)$ multiplied by above derived constrictivity $(\delta)$ (Eq. 15)

$$
\varepsilon_{\mathrm{eff}}=\delta \varepsilon_{\mathrm{tot}}
$$

As presented earlier (Figure 8a), the constrictivity $\delta_{\text {elc }}$ of the two sintered diaphragm series shows a positive correlation with the total porosity $\varepsilon_{\text {tot }}$. Hence, the effective porosity $\left(\varepsilon_{\text {eff }}\right)$ is increasing with increasing $\varepsilon_{\text {tot }}$ (Table 1, Figure $8 \mathrm{~b}$ ). The trend lines can be derived from Archie's law and the combination of Eqs. 13 and 15 give a power law dependency between the effective and the total porosity (Eq. 16)

$$
\varepsilon_{\mathrm{eff}}=\tau_{\mathrm{geo}} \varepsilon_{\mathrm{tot}}^{\mathrm{m}}
$$

Therefore, if constrictivity is not known, the Archie's law together with the measured geometric tortuosity can be used
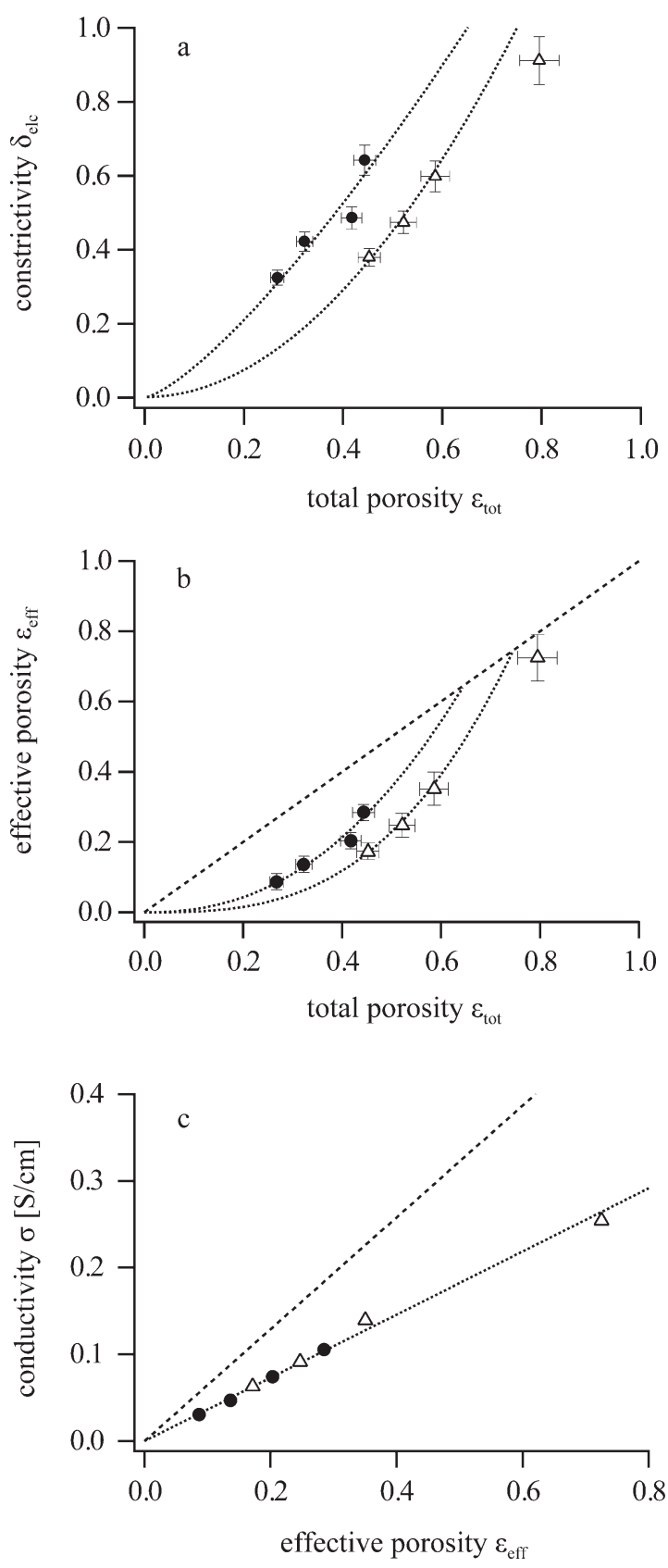

Figure 8. (a) Constrictivity $\delta_{\text {elc }}$ vs. the total porosity $\varepsilon_{\text {tot }}$ (filled circles for olivine, open triangles for wollastonite).

The error bars indicate $5.0 \%$ of the $\varepsilon_{\text {tot }}$ values (estimated) and $7.1 \%$ of the $\delta_{\text {elc }}$ values (calculated according to Eq. 14). The dotted trend lines are fitted according to Archie's law ${ }^{1}$. (b) Effective porosity $\varepsilon_{\text {eff }}$ vs. total porosity $\varepsilon_{\text {tot }}$ of the diaphragms series. The error bars indicate $5.0 \%$ of the $\varepsilon_{\text {tot }}$ values (estimated) and $8.7 \%$ of the $\varepsilon_{\text {eff }}$ values (calculated according to Eq. 17). (c) Conductivity $\sigma$ vs. effective porosity $\varepsilon_{\text {eff }}$ of the investigated diaphragm series.

for the calculation of $\varepsilon_{\mathrm{eff}}$ for the whole porosity range. The pronounced increase of $\varepsilon_{\text {eff }}$ with increasing $\varepsilon_{\text {tot }}$ can be explained with the fact that the constrictions of the pore pathways diminish with higher porosities and an increasing fraction of the pore space is effectively used for mass 
transport through the diaphragms. However, it should be noted that $\varepsilon_{\text {eff }}$ cannot exceed the total porosity.

The effective porosity can be considered as a corrected total porosity that takes into account the effect of pore pathway constrictions. Geometrically, it is the sum of all crosssectional areas of the pore path constrictions, multiplied with the length of the pore path $\left(l_{\text {eff }}\right)($ Eq. 17)

$$
\varepsilon_{\mathrm{ef} f}=\sum \frac{\pi}{4} r_{\min }^{2} * l_{\mathrm{eff}}
$$

With the introduction of the effective porosity, the pore structure model is simplified so that the effective conductivity is simply a function of the effective porosity over the geometric tortuosity. Following Eqs. 8 and 16, a linear relation between the measured ion conductivities $\left(\sigma_{\text {eff }}\right)$ and the effective porosity can be derived. In contrast to Eq. 8, which expresses the power law dependency between $\sigma_{\text {eff }}$ and $\varepsilon_{\text {tot }}$, the ion conductivity is independent from the series specific $m$ factors (Eq. 18)

$$
\frac{\sigma_{\mathrm{eff}}}{\sigma_{0}}=\frac{\varepsilon_{\mathrm{eff}}}{\tau_{\mathrm{geo}}}
$$

Consequently, data points of both diaphragm series lie on the same line (Figure $8 \mathrm{c}$ ), and the slope of the line is given by the almost constant geometric tortuosity of $\tau_{\text {geo }}=1.77$. The fact that all data points define a single trend line, although they represent two materials with different particle shapes (isometric olivine and fibrous wollastonite) and pores structure indicates that the ratio of effective porosity over tortuosity captures all microstructure features, which are relevant for the transport process under investigation.

\section{Conclusions}

In this study, the effect of pore structure on macroscopic transport properties of porous diaphragms is investigated on the basis of synchrotron $\mathrm{x}$-ray absorption tomography and electrical impedance spectroscopy. New possibilities arise from developments in 3-D image modelling, which allow for the quantification of transport relevant topological features such as tortuosity $(\tau)$, porosity $(\varepsilon)$, PSDs, and constrictivity $(\delta)$. To our knowledge, it is the first time that the latter parameter has been determined for an entire complex pore network.

In summary, it can be concluded that porosity alone cannot account for all pore structure effects in the investigated mineral diaphragms. Because olivine (isometric) and wollastonite (fibrous) exhibit different particle morphologies, related differences in diaphragm pore structures lead to distinct mass transport capabilities of the two diaphragm series. The respective trend lines can be described with the help of Archie's law, which results in a specific power law parameter $m$ for each sample series. Based on the empirically derived $m$-factor, extrapolations of the macroscopic transport properties can be made for the whole porosity range.

For both investigated series, the geometric tortuosity is nearly constant over a wide range of porosities and hence it is not discriminating the different pore structures and the corresponding transport properties. In contrast, the present investigation reveals strong evidence that the pore structure effects are dominated to a large degree by the size of pore neck constrictions. In the presented study, the bottleneck effect is described in two ways: (1) by direct measurement of the $r_{\min }$ and $r_{\max }$, which leads to the constriction factor $(\beta)$ and $(2)$ by indirect calculation of the constrictivity $(\delta)$ from EIS measurements. The relation between the constriction factor $(\beta)$ and constrictivity is published elsewhere. ${ }^{24}$

The introduction of geometric parameters that are measured directly from tomographs opens new possibilities to study pore structure effects. Using an extended pore structure model that includes the microscopic constrictivity together with tortuosity and porosity, the macroscopic conductivity can be calculated precisely with data that is extracted exclusively and directly from tomographs.

The good match between measured and calculated ion conductivities indicates the validity of the new methods for microstructure quantification. In our current research projects, these methods are further improved to use them for the fundamental study of transport in a wide range of porous media such as fuel cell electrodes, host rocks for radioactive waste disposal, reservoir rocks for natural gas and oil, moisture transport in concrete, and gas separation diaphragms in alkaline electrolysis cells.

\section{Acknowledgments}

This work was carried out within the framework of the Swiss Federal Commission for Technology and Innovation (CTI, project no. 8574.2 PFIW-IW). Financial supports from Industry High Technology Ltd. (IHT), the state of Switzerland, and the University of Fribourg (Switzerland) are gratefully acknowledged. The study was in part supported by Centre d'Imagerie BioMédicale (CIBM) of the UNIL, UNIGE, HUG, CHUV, EPFL, and the Leenaards and Jeantet Foundations.

\section{Literature Cited}

1. Archie GE. The electrical resistivity $\log$ as an aid in determining some reservoir characteristics. Trans AIME. 1942;146:54-62.

2. Barrande M, Bouchet R, Denoyel R. Tortuosity of porous particles. Anal Chem. 2007;79:9115-9121.

3. Carman PC. Flow of Gases Through Porous Media. London: Butterworths, 1956.

4. Engblom SO, Myland JC, Oldham KB, Taylor AL, Topic WC. Electrochemical detection of large channels in porous rocks. J Appl Electrochem. 2003;33:51-59.

5. Garcia-Gabaldon M, Perez-Herranz V, Sanchez E, Mestre S. Effect of porosity on the effective electrical conductivity of different ceramic membranes used as separators in electrochemical reactors. J Membr Sci. 2006;280:536-544.

6. Kozeny J. Über kapillare Leitung des Wassers im Boden. Akad Wiss Wien. 1927;136:217-306.

7. Myland JC, Oldham KB, Schiewe J, Taylor AL. Electrochemical study of the pore structure of sandstone and similar media. Can J Chem. 1998;76:1688-1694.

8. Oldham KB, Topol LE. Electrochemical investigations of porous media. I. Theory of potentiostatic methods. $J$ Phys Chem. 1967;71:3007-3013.

9. Wyllie MR, Rose WD. Application of the Kozeny equation to consolidated porous media. Nature. 1950; 165:972.

10. Dullien FAL. Porous Media, Fluid Transport and Pore Structure. San Diego: Academic Press Limited, 1992. 
11. Blake FC. The resistance of packing to fluid flow. Trans Am Inst Chem Eng. 1922;14:415-421.

12. Petersen EE. Diffusion in a pore of varying cross section. AIChE J. 1958;4:343-345.

13. Fievet P, Szymczyk A, Aoubiza B, Pagetti J. Evaluation of three methods for the characterisation of the membrane-solution interface: streaming potential, membrane potential and electrolyte conductivity inside pores. $J$ Membr Sci. 2000;168:87-100.

14. Stojadinovic J, Wiedenmann D, Gorbar M, La Mantia F, Suarez L, Zakaznova-Herzog V, Vogt UF, Grobéty B, Züttel A. Electrochemical characterization of porous gas separation diaphragms. ECS Electrochem Lett. 2012;1:F25-F28.

15. Stampanoni M, Groso A, Isenegger A, Mikuljan G, Chen Q, Meister D, Lange M, Betemps R, Henein S, Abela R. TOMCAT: a beamline for TOmographic Microscopy and Coherent rAdiology experiments. AIP Conference Proceedings. Sync Rad Instr. 2007:879:848-851.

16. Holzer L, Indutnyi F, Gasser P, Münch B, Wegmann M. Three-dimensional analysis of porous $\mathrm{BaTiO} 3$ ceramics using FIB nanotomography. J Microsc. 2004;216:84-95.

17. Münch B, Holzer L. Contradicting geometrical concepts in pore size analysis attained with electron microscopy and mercury intrusion. J Am Ceram Soc. 5008;91:40594067.

18. Diamond S. Mercury porosimetry: an inappropriate method for the measurement of pore size distributions in cement based materials. Cem Concr Res. 2000;30(10):1517-1525.

19. Holzer L, Iwanschitz B, Hocker Th, Münch B, Prestat M, Wiedenmann D, Vogt U, Holtappels P, Sfeir J, Mai A, Graule Th. Microstructure degradation of cermet anodes for solid oxide fuel cells: quantification of nickel grain growth in dry and in humid atmospheres. $J$ Power Sources. 2011;196:1279-1294.

20. Lindquist WB, Lee SM, Coker DA, Jones KW, Spanne P. Medial axis analysis of void structure in three-dimensional tomographic images of porous media. $J$ Geophys Res. 1996;101:8297-8310.

21. Jungnickel D. Graphs, Network and Algorithmus. Berlin: Springer, 1999.

22. Cormen TH, Stein C, Leiserson CE, Rivest RL. Introduction to Algorithms. Cambridge, MA: MIT Press, 2009.

23. Comiti J, Renaud M. A new model for determining mean structure parameters of fixed beds from pressure drop measurements: applications to beds packed with parallelepipedal particles. Chem Eng Sci. 1998;44:15391545.

24. Holzer L, Wiedenmann D, Münch B, Keller L, Prestat M, Gasser P, Robertson I, Grobéty B. Quantitative description of bottlenecks in 3D-microstructures: a heuristic approach to calculate effective transport properties. J Mater Sci. 2013;48:2934-2952. 